\title{
Técnica quirúrgica de la implantación de dispositivo ajustable transobsturador anti-incontinencia urinaria masculina tipo Atoms en una clínica de III nivel en Bogotá
}

\section{Surgical Technique of the Implantation of Adjustable Masculine Transobsturador Anti-Incontinence Urinary Device Type Atoms in a Clinic of III Level in Bogotá}

\author{
Jairo Humberto Correa ${ }^{1}$ Jhonatan Sair Santafé $G^{1}$ \\ ${ }^{1}$ Fundación Universitaria Sanitas, Bogotá, Colombia \\ Urol Colomb 2019;28:100-105.
}

\section{Introducción}

La prevalencia de incontinencia urinaria de esfuerzo (IUE) post-prostatectomía varía entre el $4 \%$ y el 50\%, siendo más frecuente en pacientes que son llevados a prostatectomía radical. Es una condición que afecta la calidad de vida de los pacientes y presenta un impacto de grado variable de severidad. Existen diferentes opciones para el tratamiento de esa entidad, dentro de los cuales se encuentran: terapia de piso pélvico con biofeedback, fisioterapia, sistemas recolectores de orina, clamps peneanos, inyecciones periuretrales de materiales abultantes, esfínter urinario artificial (EUA) y cintas pubouretrales. ${ }^{1,2}$ El gold standard para la IUE es el EUA con tasa de éxito entre $50 \%-80 \%$, no obstante, con una tasa de explante de hasta el $33 \%$. Se han desarrollado otras alternativas con cintas pubouretrales las cuales han reportado una tasa de éxito hasta del $83 \%{ }^{2}$ La principal limitante de esas alternativas en el manejo de la IUE, ha sido la necesidad de reajustar esos dispositivos con procedimientos quirúrgicos, exponiendo en repetidas ocasiones a los pacientes a los riesgos de anestesia y del procedimiento quirúrgico, situación que se ha tratado de mejorar con el dispositivo ATOMS $®$, introducido en Europa desde marzo del 2009, el cual ofrece la oportunidad de realizar tales ajustes en el consultorio. ${ }^{3}$ La indicación para la colocación del ATOMS $®$ es la IUE, sin tener claridad en su severidad, hasta en un 92,9\% de las ocasiones, y usualmente son pacientes con antecedente de cirugías previas antiincontinencia fallidas (34,3\%). El tiempo quirúrgico promedio es de 47 minutos, entre 29 minutos a 112 minutos. ${ }^{1}$
Address for correspondence Jairo Humberto Correa, MD, Fundación Universitaria Sanitas, Bogotá, Colombia (e-mail: betoco@hotmail.com).

El sistema anti-incontinencia ajustable transobturador masculino (ATOMS $®$ ) es una cinta que tiene como mecanismo la compresión no circunferencial suburetral a nivel de uretra bulbar, y tiene la característica de ser ajustable con el objetivo de evitar las reintervenciones quirúrgicas. Ese dispositivo, al contrario del EUA, no compromete el plexo venoso uretral, lo cual disminuye el riesgo de perforación o atrofia uretral, y el abordaje quirúrgico para su instalación es mínimamente invasivo. ${ }^{4}$ El dispositivo tiene 2 componentes principales: una malla (cabestrillo) de polipropileno macroporosa monofilamento con un cojinete ajustable de silicona con sutura monofilamento para fijación de la malla y un puerto de titanio recubierto de silicona de grado médico, el cual permite la manipulación para el reajuste en consultorio según sea requerido. ${ }^{5}$

En 2012, Hoda y col., realizan el reporte de su experiencia en el manejo de la IUE post-prostatectomía mediante el uso del ATOMS ${ }^{\circledR}$. Colocan entonces el dispositivo en 99 pacientes entre 2009 a 2010, mediante el uso de la técnica out-in, a través del foramen obturador y anclando el dispositivo en la rama inferior del pubis, luego dejan el puerto de titanio subcutáneo en la región izquierda de la sínfisis para realizar ajustes vía ese puerto. Se excluyen aquellos pacientes con hiperactividad del detrusor, estrechez uretral o de la anastomosis uretrovesical, y progresión del PSA (antígeno prostático específico) en el último año. Intervienen pacientes con IUE en diferentes grados de severidad: uso de 1-2 pañales/protectores cada día (IUE leve), 3-5 pañales/ protectores diarios (IUE moderada) y aquellos con más de 5 pañales/protectores diarios (IUE severa). ${ }^{1}$ Esa clasificación es
DOI https://doi.org/ $10.1055 / \mathrm{s}-0039-1688754$. ISSN 0120-789X. eISSN 2027-0119.
Copyright (c) 2019, Sociedad Colombiana License terms de Urología. Publicado por Thieme Revinter Publicações Ltda., Rio de Janeiro, Brazil. Todos los derechos reservados. 
usada en nuestro servicio para clasificar, evaluar la mejoría y realizar el seguimiento a nuestros pacientes con IUE.

La técnica quirúrgica de la implantación del ATOMS® ha mantenido un esquema similar, con algunas modificaciones realizadas por algunos autores en centros de referencia con el fin de mejorar la tasa de complicaciones por infección del sitio quirúrgico o el dolor postoperatorio. ${ }^{1,5,6}$ La técnica quirúrgica usada en el estudio más grande de Hoda y col., se describe como un procedimiento bajo anestesia general, en posición de litotomía, se avanza sonda uretral $16 \mathrm{Fr}$, se realiza una incisión mediana de $5 \mathrm{~cm}$ vertical en periné por debajo del borde inferior de la sínfisis púbica para exponer el músculo bulboesponjoso, se diseca cada triángulo de la aponeurosis perineal delimitado lateralmente por músculos isquiocavernosos. A través de una incisión sobre la fascia pélvica expuesta, se expone el músculo obturador bajo la rama ósea isquiopúbica. La colocación del dispositivo se realiza mediante la técnica out-in a través del foramen obturador, los extremos de la cinta emergen y se suturan en la parte central al cojinete. En ese punto de la intervención, se realiza el cambio de guantes estériles e instrumental quirúrgico, se coloca el puerto de titanio que se ubica profundo en el espacio celular subcutáneo en la región izquierda de la sínfisis del pubis y se fija con 2 suturas. Se instilan $6 \mathrm{~mL}$ de medio de contraste + solución salina (1:1). Se cierra la incisión perineal sin dejar algún tipo de dren y la sonda uretral se retira en 24 horas antes del egreso. ${ }^{1}$ Mencionan, que ante la necesidad de retiro de un dispositivo anti-incontinencia previo, la colocación del ATOMS ${ }^{\circledR}$ se posterga a 3 meses. Por otra parte, Bauer y col., describe la colocación del sistema en todos los grados de incontinencia, incluyendo quienes hayan recibido radioterapia, con reporte de mejoría de la IUE hasta en un $83 \%$, y describen que en caso de requerir retiro de un dispositivo anti-incontinencia previo se realiza en el mismo tiempo quirúrgico. Anotan que se realiza bajo anestesia general o raquídea, en posición de litotomía, antibiótico endovenoso profiláctico, uso de sonda uretral $14 \mathrm{Fr}$, cobertura del glande con una compresa estéril para evitar secreciones que puedan contaminar el área quirúrgica. Realizan incisión vertical de $5 \mathrm{~cm}$ en el periné, exponiendo el músculo bulboesponjoso. Describen el uso de un retractor para mejorar la exposición de la incisión perineal, se diseca ambas fosas isquiorrectales, con el dedo índice se comprueba los forámenes obturadores. Hasta este momento, describen debe destaparse el dispositivo ATOMS ${ }^{\circledR}$ de su envoltorio, acomodan la cinta verificando la correcta orientación para posteriormente con la técnica outside-in, a través del foramen obturador, pasar cada extremo de la cinta hasta el cojinete del dispositivo. Posteriormente, verificada la orientación adecuada de la cinta, se realiza la tracción de ambos extremos de la cinta para acomodar el dispositivo en su posición suburetral, y se anuda para fijar usando la sutura monofilamento de polipropileno en el cojinete del dispositivo. Se realiza una incisión lateral izquierda a la sínfisis del pubis cerca a la base del pene, con ayuda de un electrobisturí monopolar hasta el tejido celular subcutáneo para tener un espacio donde el puerto de titanio será ubicado, también se puede realizar con ayuda de un tunelizador recto. Se realiza el lavado de heridas con solución yodada, se puede realizar el ajuste del cojinete con una jeringa de $10 \mathrm{~mL}$ a través del puerto, en promedio entre 4 a $8 \mathrm{~mL}$, y un $\mathrm{mL}$ adicional en casos de incontinencia moderada a severa, y finalmente se realiza el cierre de piel por planos, con cierre de 3 planos en el periné. ${ }^{6}$

Cuatro cambios se consideran que han marcado la diferencia en la mejoría de la técnica quirúrgica para la colocación del dispositivo anti-incontinencia ajustable transobturador masculino: 1) la apertura del dispositivo justo después de haber expuesto la zona quirúrgica donde se fijará el dispositivo, 2) el cambio de guantes en el momento de instalar el puerto de titanio, 3 ) el ajuste del cojinete en región suburetral y la fijación inmediata de los extremos de la cinta, y 4) la realización de la ubicación y fijación del puerto de titanio subdartos sin incisiones adicionales. ${ }^{6,7}$ Nuestra técnica quirúrgica es basada en la descripción original, con las modificaciones que han demostrado una disminución en la tasa de complicaciones en la inserción del dispositivo ATOMS $\circledast$.

La evaluación de los pacientes con IUE que son llevados a colocación de ATOMS ${ }^{\circledR}$ previo al egreso, se realiza mediante uroflujometría, medición ecográfica del residuo potmiccional y evaluación del dolor pélvico mediante la escala visual análoga (VAS). La evaluación preoperatoria y postoperatoria usualmente se realiza a través del examen físico, pad test de 24 horas y conteo del uso de protectores durante 24 horas, asociado usualmente con un cuestionario de calidad de vida validado. ${ }^{1,7}$ En nuestra institución se egresa al paciente sin sonda y previa comprobación de micción espontánea. Si presenta retención urinaria se vuelve a colocar sonda Foley 16 Fr 2 vías y en el control postoperatorio y a las 2 semanas se retira la sonda para probar nuevamente la micción espontánea. No hemos tenido nuevos episodios de retención urinaria después de ese primer control. Los controles se realizan a cada 4 semanas durante los 3 primeros meses.

Se ha descrito la necesidad de realización de los reajustes cada 6 meses con $2 \mathrm{~mL}$ de solución salina hasta lograr resultados deseados. ${ }^{1}$ Se ha reportado un promedio de 3,8 ajustes para obtener el resultado deseado (1-6 ajustes): sequedad, mejoría y/o satisfacción. No obstante, sigue existiendo un déficit en la literatura científica que permita evaluar un seguimiento a largo plazo, se ha descrito la evaluación de resultados post quirúrgicos durante un periodo entre 12-33 meses, con una tasa de éxito entre $81.6 \%$ a $92 \%{ }^{1,7}$ satisfacción global de $93.2 \%$ y una disminución promedio del uso de pañal o protectores de 7.1 unidades/24 horas hasta 1.3 unidades/24 horas, con un valor estadísticamente significativo. ${ }^{1}$ En nuestra experiencia, realizo el primer ajuste, cuando es necesario, a las 4 semanas.

Dentro de las complicaciones postoperatorias descritas, se encuentran algunas transitorias como la retención urinaria, Hoda y col., reporta hasta del 2\% que resuelve con retiro de $4 \mathrm{~mL}$ del cojinete y permanencia de la sonda uretral por 48 horas; las disestesias perineales o escrotales o dolor se ha descrito de hasta $68,7 \%$, no obstante, resuelve a las 3-4 
semanas sin necesidad de medicamentos opioides. No se han reportado lesiones uretrales, vesicales 0 erosiones secundarias con el dispositivo transobturador ajustable, y en series de casos donde ha habido alguna de esas, se asocian con retiro de otros dispositivos anti-incontinencia o de EUA. ${ }^{1,7}$ La tasa de infección del sitio operatorio oscila hasta el $4 \%$, predominantemente comprometiendo el sitio del puerto para los ajustes de la cinta anti-incontinencia, Hoda y col. describe esos dispositivos fueron retirados y reimplantados exitosamente 3 meses después de finalizar el tratamiento antibiótico. ${ }^{1}$

\section{Técnica Quirúrgica}

Paso de sonda Foley 16 Fr 2 vías y evacuación de vejiga ( - Fig. 1).

Reparos anatómicos: 1) Agujeros obturadores, 2) bordes inferiores de ramas isquiopúbicas, 3) Rafé médio perineal (-Fig. 2).

Disección de los músculos bulboesponjosos (-Fig. 3).

Lavado de lecho con solución antibiótica de Gentamicina $160 \mathrm{mg}$ en $250 \mathrm{~mL}$ de suero fisiológico (-Fig. 4).

Preparación del dispositivo. Se anuda extremo del polipropileno de la malla en pasador helicoidal reutilizable (-Figs. 5 years $\mathbf{6}$ ).

Se palpa orificio obturador y se perfora la membrana obturatriz hasta la fosa obturadora (-Fig. 7).

Se avanza el pasador helicoidal, sosteniendo el mango en plano horizontal y exteriorizando la punta de la aguja a través del plano formado entre borde lateral del cuerpo cavernoso y el diafragma urogenital en sentido out-in. Se avanza en la malla contralateral con los mismos reparos anatómicos en espejo (-Fig. 8).

Se orientan las mallas sin torsión sobre su eje longitudinal y se traccionan con firmeza (-Fig. 9).

Ubicación del dispositivo caudal al músculo bulboespongioso ( $\mathbf{- F i g . ~ 1 0 )}$.

Se anudan suturas de polipropileno a través de orificios de la malla proximal y distalmente, se anudan firmemente y se corta extremo de la malla a $1 \mathrm{~cm}$ del nudo más distal. Procedimiento bilateral (-Fig. 11). En figura 12 se muestra como debe quedar los extremos de las mallas en forma de " $\mathrm{X}$ " (-Fig. 12).

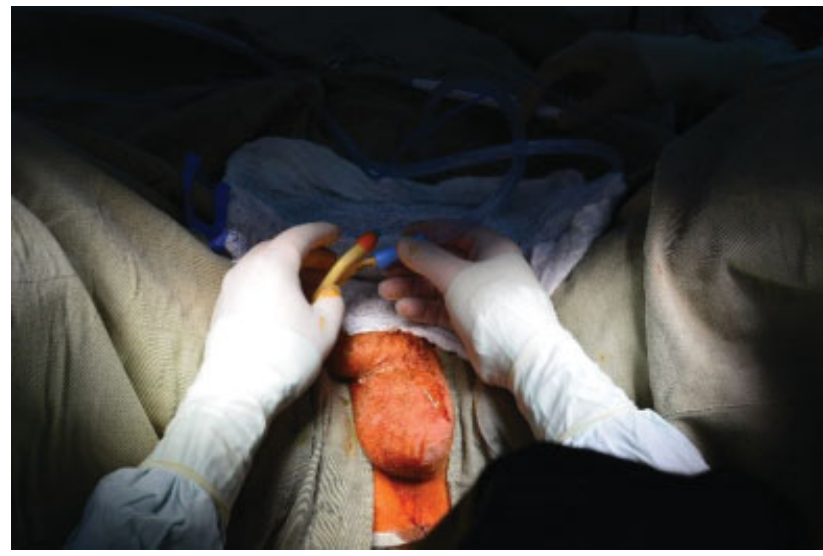

Fig. 1 Paso de sonda Foley 16 Fr 2 vías y evacuación de vejiga.

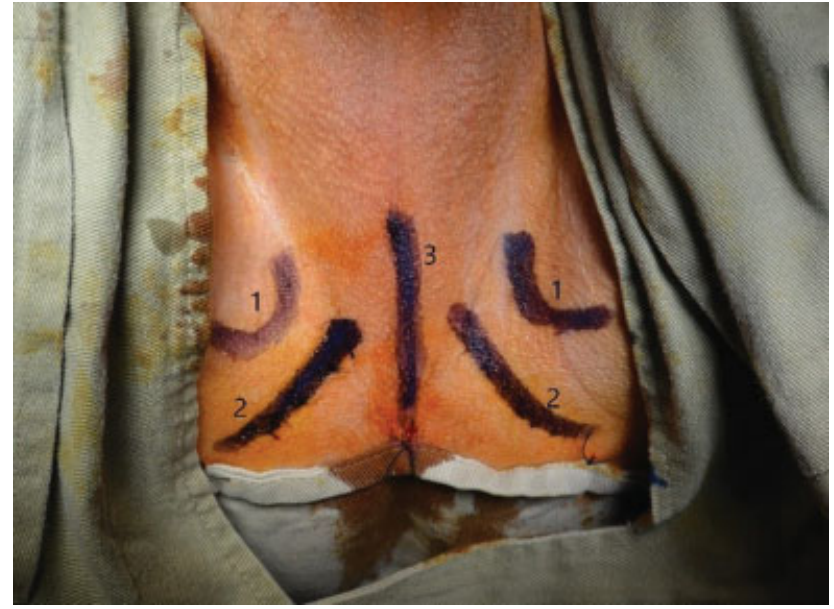

Fig. 2 Reparos anatómicos: 1) Agujeros obturadores, 2) bordes inferiores de ramas isquiopúbicas, 3) Rafé médio perineal.

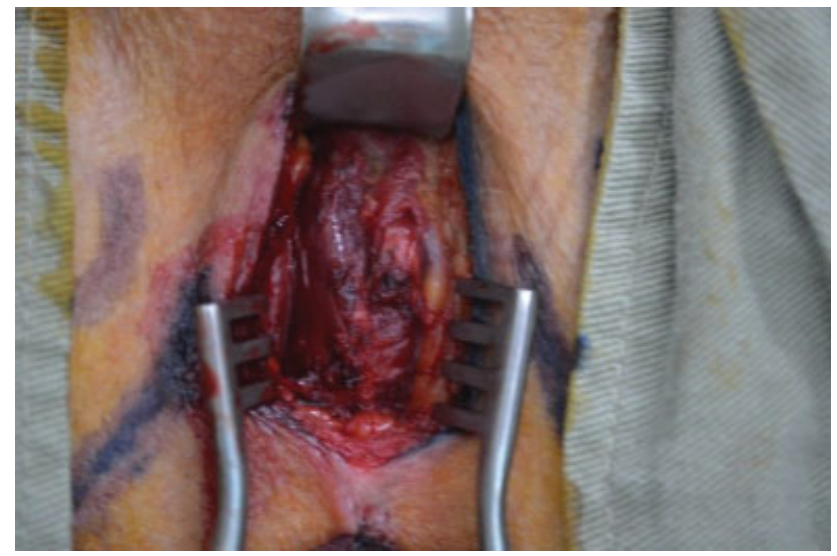

Fig. 3 Disección de los músculos bulboesponjosos.

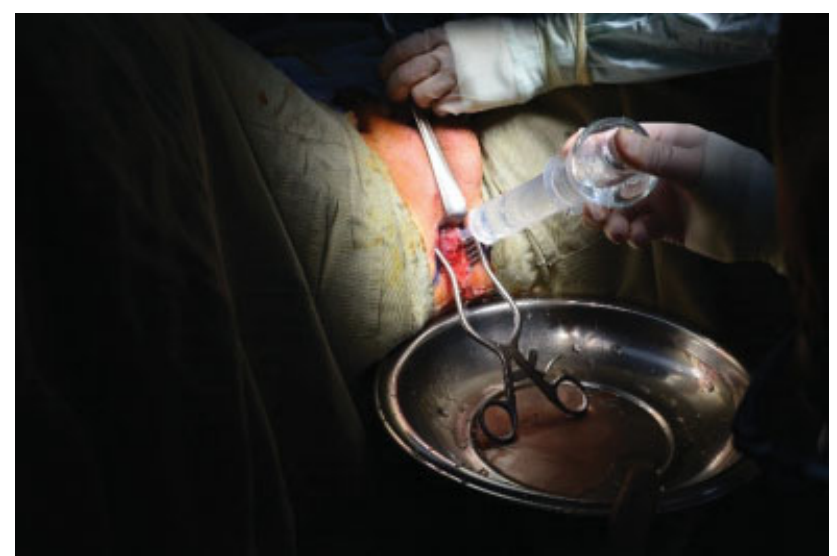

Fig. 4 Lavado de lecho con solución antibiótica de Gentamicina $160 \mathrm{mg}$ en $250 \mathrm{~mL}$ de suero fisiológico.

Se purga cojín con $10 \mathrm{~mL}$ de suero fisiológico, puncionando membrana de silicona del puerto de ajuste $\mathrm{y}$ se permite retorno espontáneo del aire y de volumen sobrante de suero fisiológico. Las propiedades elásticas del cojín de silicona del ATOMS ${ }^{\circledR}$ retornan a la jeringa unos $3 \mathrm{~mL}$ en promedio de suero fisiológico. Cuando cesa el retorno, se 


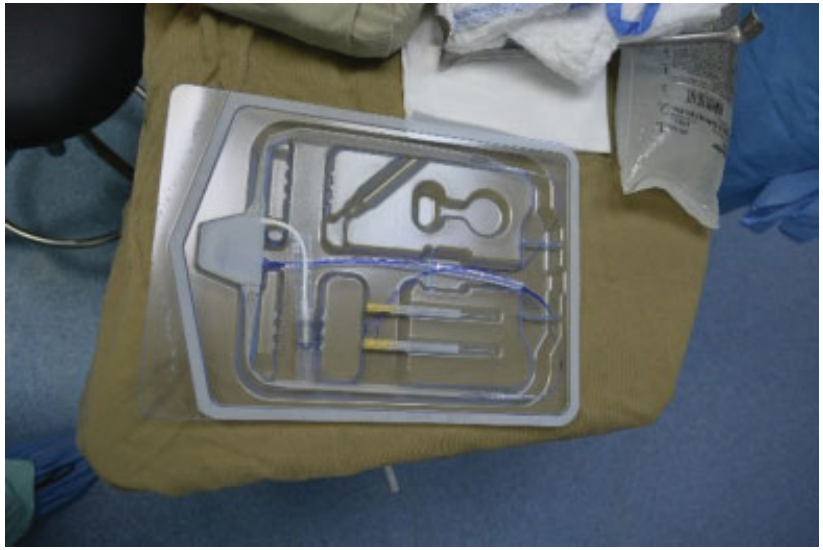

Fig. 5 Preparación del dispositivo. Se anuda extremo del polipropileno de la malla en pasador helicoidal reutilizable.

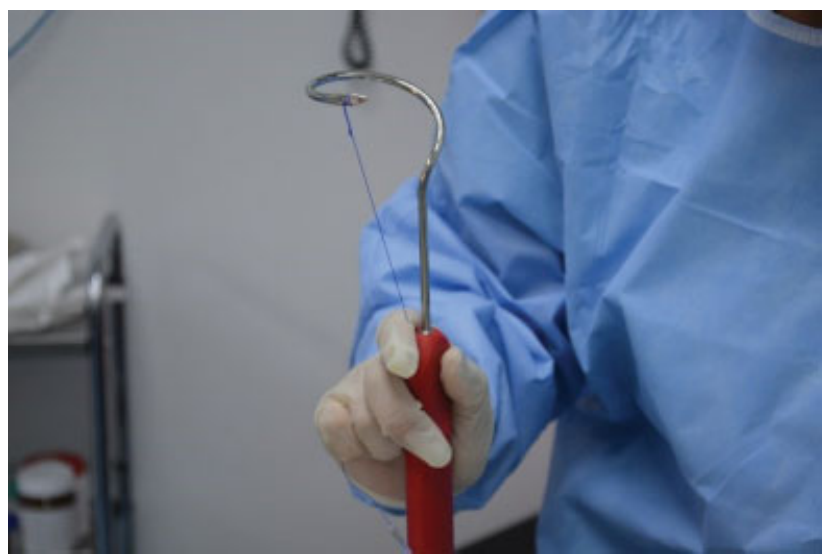

Fig. 6 Preparación del dispositivo. Se anuda extremo del polipropileno de la malla en pasador helicoidal reutilizable.

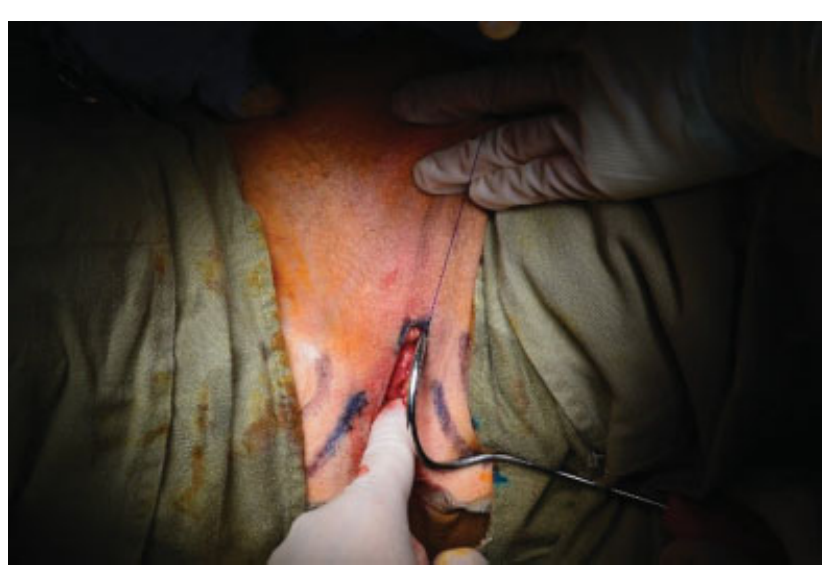

Fig. 7 Se palpa orificio obturador y se perfora la membrana obturatriz hasta la fosa obturadora.

adicionan $2 \mathrm{~mL}$ de suero fisiológico y con este volumen se deja ajustado el dispositivo (-Figs. 13 y 14).

Cambio de guantes de todo el personal quirúrgico. Se realiza túnel subdartos escrotal izquierdo y se posiciona el puerto de ajuste de titanio cubierto con silicona en ese bolsillo (-Fig. 15).

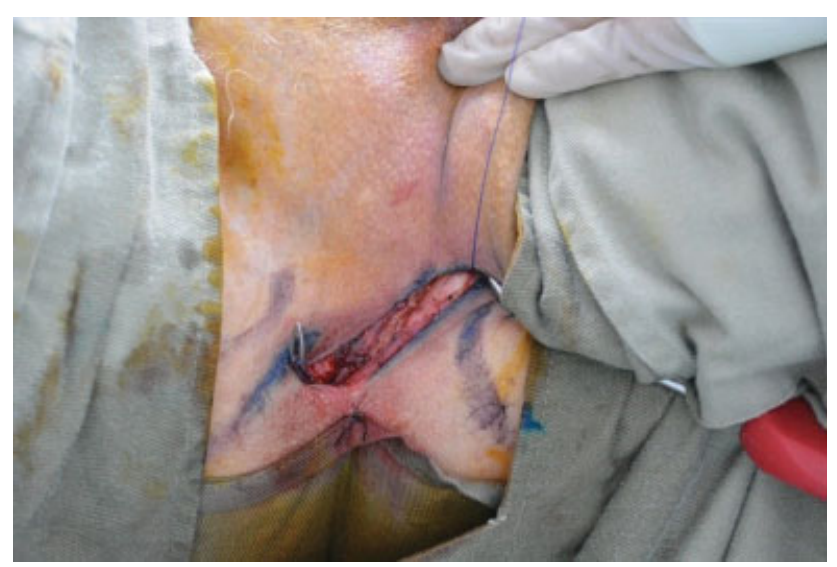

Fig. 8 Se avanza el pasador helicoidal, sosteniendo el mango en plano horizontal y exteriorizando la punta de la aguja a través del plano formado entre borde lateral del cuerpo cavernoso y el diafragma urogenital en sentido out-in. Se avanza en la malla contralateral con los mismos reparos anatómicos en espejo.

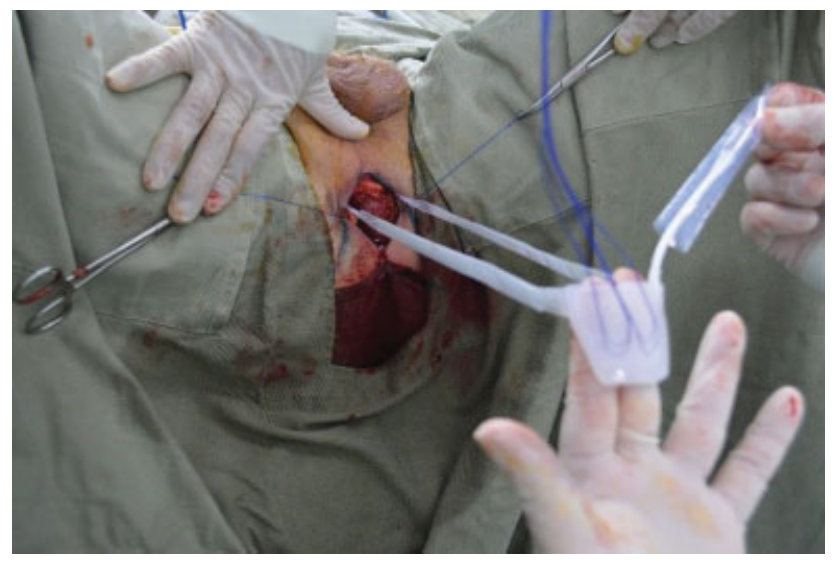

Fig. 9 Se orientan las mallas sin torsión sobre su eje longitudinal y se traccionan con firmeza.

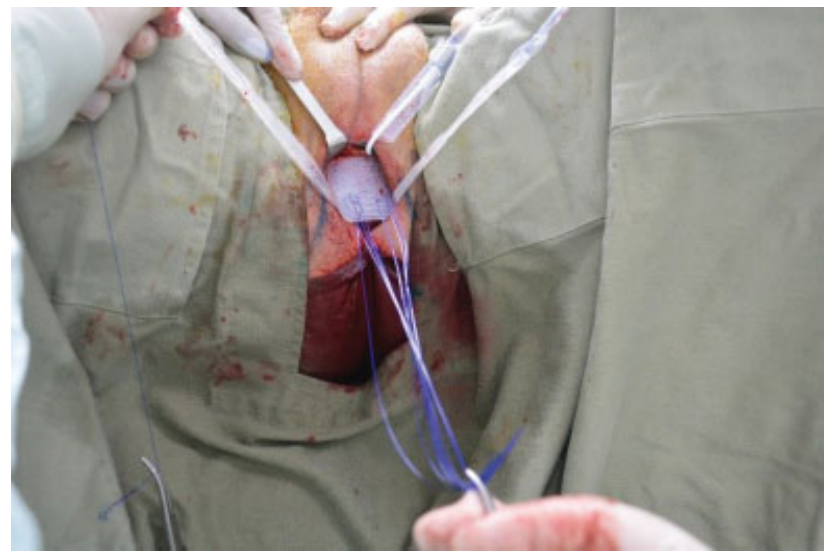

Fig. 10 Ubicación del dispositivo caudal al músculo bulboespongioso.

Se cierra el cuello del túnel subdartos con sutura absorbible para evitar la migración del puerto de ajuste (-Fig. 16).

Cierre de incisión quirúrgica en 3 planos con sutura absorbible (-Fig. 17).

Revista Urología Colombiana / Colombian Urology Journal Vol. 28 No. 2/2019 
Técnica quirúrgica de la implantación de dispositivo ajustable transobsturador anti-incontinencia urinaria masculina

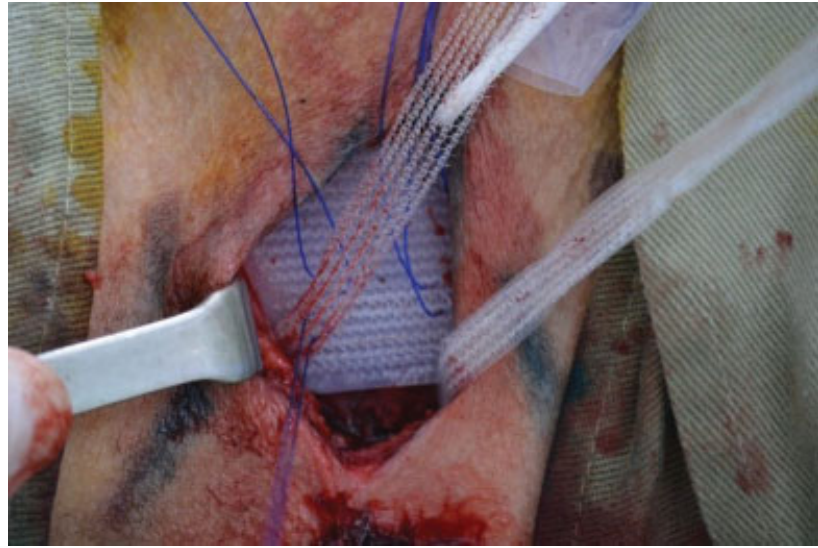

Fig. 11 Se anudan suturas de polipropileno a través de orificios de la malla proximal y distalmente, se anudan firmemente y se corta el extremo de la malla a $1 \mathrm{~cm}$ del nudo más distal. Procedimiento bilateral.

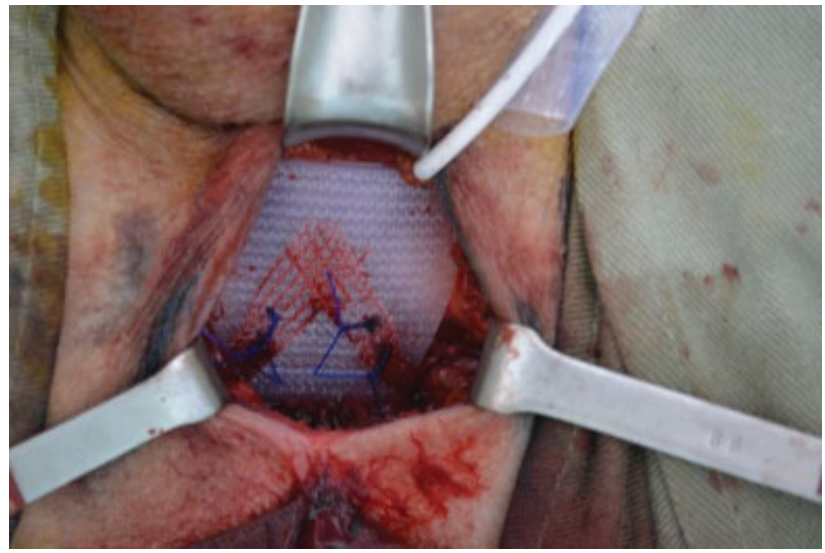

Fig. 12 En la figura se muestra cómo deben quedar los extremos de las mallas en forma de "X."

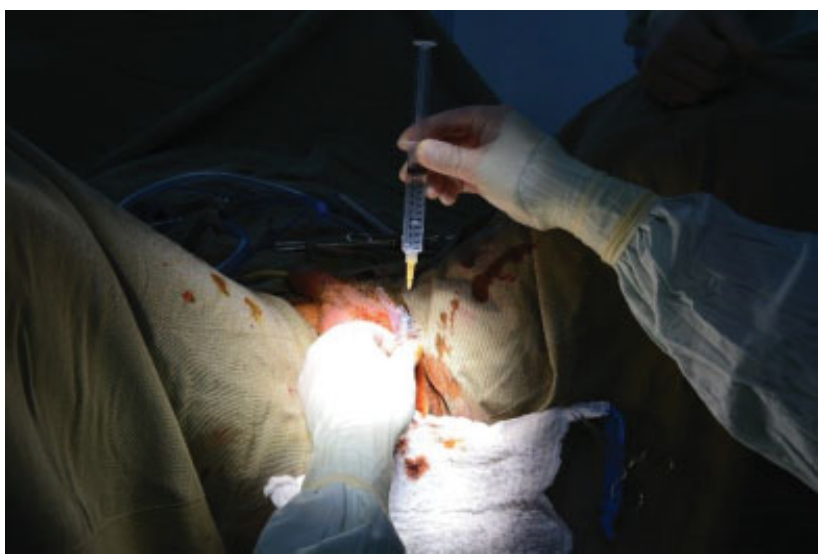

Fig. 13 Se purga el cojín con $10 \mathrm{~mL}$ de suero fisiológico, puncionando membrana de silicona del puerto de ajuste y se permite el retorno espontáneo del aire y del volumen sobrante del suero fisiológico.

\section{Conclusión}

El ATOMS $®$ es un dispositivo anti-incontinencia masculina seguro y eficaz, recientemente introducido en nuestro país. La mayor experiencia viene de centros europeos, mostrando la

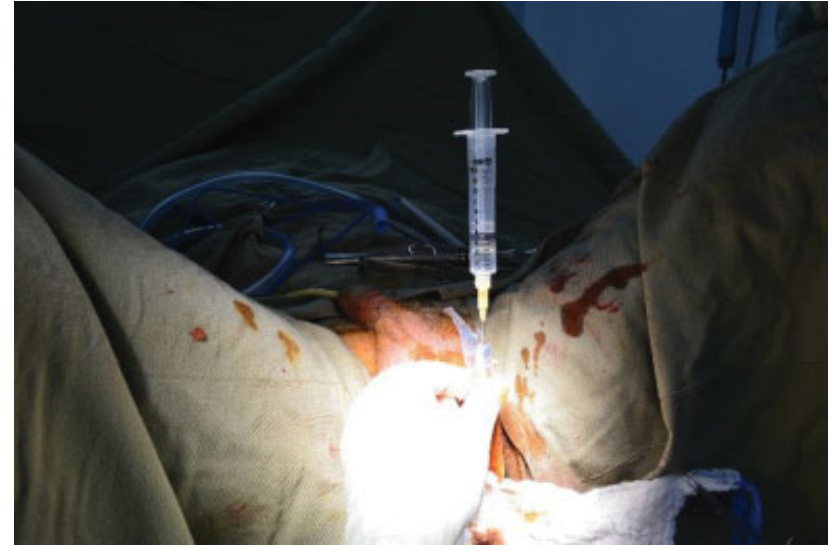

Fig. 14 Las propiedades elásticas del cojín de silicona del ATOMS $®$ retornan a la jeringa unos $3 \mathrm{~mL}$ en promedio de suero fisiológico. Cuando cesa el retorno, se adicionan $2 \mathrm{~mL}$ de suero fisiológico y con ese volumen se deja ajustado el dispositivo.

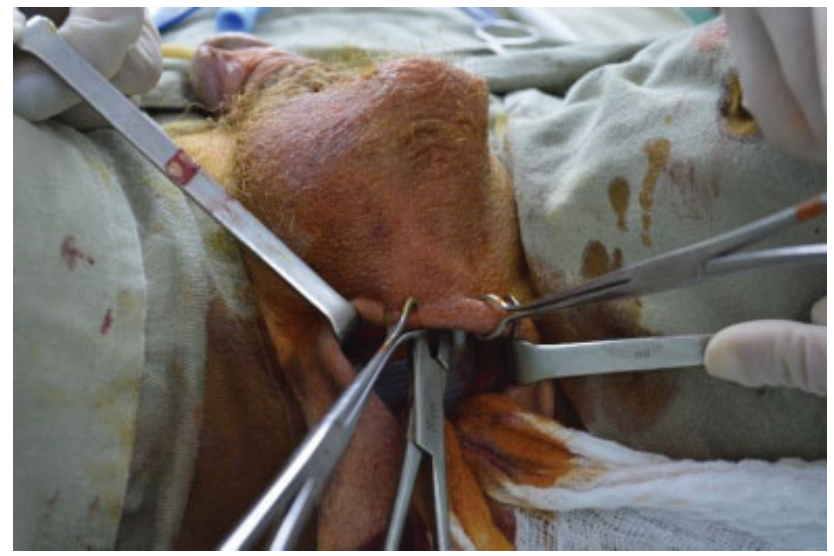

Fig. 15 Cambio de guantes de todo el personal quirúrgico. Se realiza el túnel subdartos escrotal izquierdo y se posiciona el puerto de ajuste de titanio cubierto con silicona en este bolsillo.

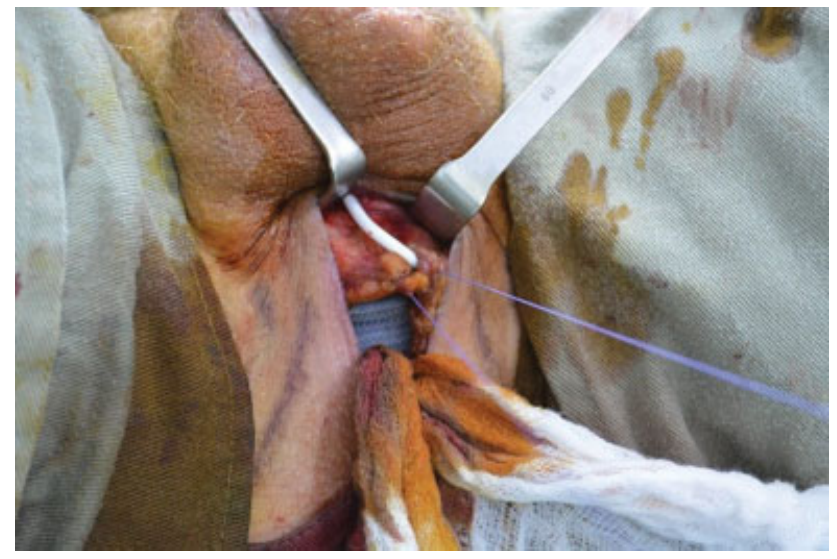

Fig. 16 Se cierra cuello del túnel subdartos con sutura absorbible para evitar la migración del puerto de ajuste.

experiencia con series de casos y hacen falta estudios bien diseñados comparando la técnica con el estándar de oro referido en las guías de manejo de la incontinencia urinaria masculina. Esos centros especializados con un alto volumen de 


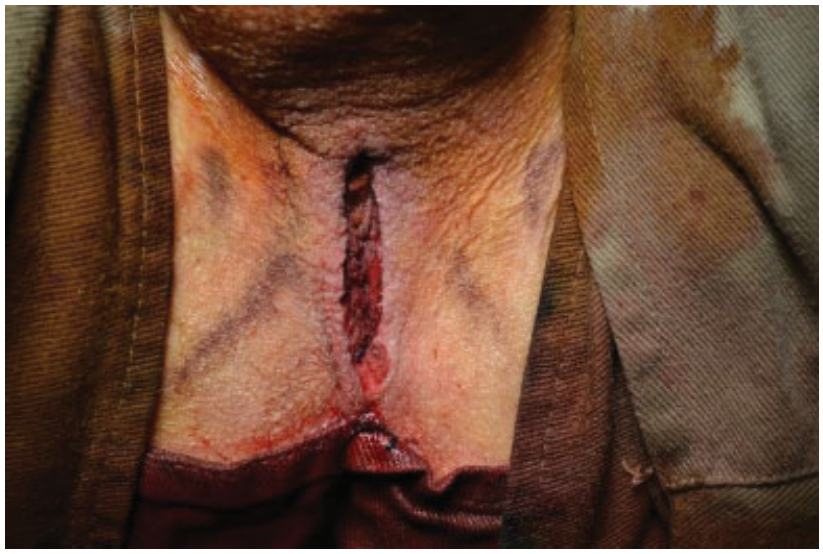

Fig. 17 Cierre de incisión quirúrgica en 3 planos con sutura absorbible.

pacientes reportan una tasa de éxito, satisfacción y mejoría similares al estándar de oro que hasta el momento continúa siendo el EUA. La técnica quirúrgica es sencilla, mínimamente invasiva, reproducible, con un entrenamiento corto y si se siguen los pasos indicados se considera un procedimiento seguro con resultados similares a los reportados en la literatura disponible. Una ventaja clara de ese dispositivo, es la posibilidad de ajustar la compresión extrínseca sobre la uretra bulbar hasta lograr los resultados deseados sin la necesidad de manipular el dispositivo por parte del paciente, logrando una micción lo más cercana posible al estado fisiológico basal.
Conflictos de interés

Los autotes declaran que no existen conflictos de interés.

\section{Bibliografía}

1 Hoda MR, Primus G, Fischereder K, et al. Early results of a European multicentre experience with a new self-anchoring adjustable transobturator system for treatment of stress urinary incontinence in men. BJU Int 2012;111:296-303

2 Burkhard FC, Bosch JLH, Lemack GE, et al. Guidelines on Urinary Incontinence. European Association of Urology; 2018

3 Van der Aa F, Drake MJ, Kasyan GR, Petrolekas A, Cornu JN. The artificial urinary sphincter after a quarter of a century: A critical systematic review of its use in male non-neurogenic incontinente. Eur Urol 2013;63:681-689

4 Krause J, Tietza S, Behrendt W, Nast J, Hamza A. Reconstructive surgery for male stress urinary incontinence: Experiences using the ATOMS ${ }^{\circledR}$ system at a single centler. GMS Interdiscip Plasrt Surg DGPM 2014;3;

5 Seweryn J, Bauer W, Ponholzer A, Schramek P. Initial experience and results with a new adjustable transobturator male system for the treatment of stress urinary incontinence. J Urol 2012; 187:956-961

6 Bauer W, Brössner C. Adjustable transobturator male system ATOMS - for the treatment of post-prostatectomy urinary incontinence: The surgical technique. 2011;30:10-16

7 Esquinas C, Arance I, Pamplona J, Moraga A, Dorado JF, Ângulo JC. Tratamiento de la incontinencia urinaria de esfuerzo tras prostatectomía con el sistema masculino transobturador ajustable (ATOMS ${ }^{\circledR}$ ) con puerto escrotal premontado. Actas Urol Esp 2018:1-10 